\title{
Laboratory and Astronomical Detection of New Carbon Chains and Rings
}

\author{
Michael C. McCarthy \\ Atomic and Molecular Physics Division, Harvard-Smithsonian Center \\ for Astrophysics, 60 Garden Street, Cambridge, MA 02138, USA
}

\begin{abstract}
Discoveries of large new organic molecules in space help provide a foundation for understanding the role of the chemical bond and organic chemistry on a cosmic scale, and by extension, an opportunity to begin to address the interesting but far more complicated questions of chemical and biological origins and the issues that relate to them. Most of the organic molecules so far detected in the interstellar gas and circumstellar shells by radio telescopes are highly unsaturated carbon chains, a configuration of linear carbon which is unstable at high density, and therefore unfamiliar on Earth. For this reason laboratory detection until quite recently has lagged behind astronomical discovery of many new carbon molecules. The application of Fourier transform microwave spectroscopy to supersonic molecular beams has now largely overcome this obstacle, yielding in just over three years the laboratory detection of forty-four new carbon chains and eight new carbon ring-chains and rings, including two rhomboidal isomers of $\mathrm{SiC}_{3}$. The set consists of 14 polyynes, 20 carbon chain radicals, and 18 free carbenes. For four other triplet chains $\mathrm{HC}_{7} \mathrm{H}, \mathrm{HC}_{9} \mathrm{H}, \mathrm{HC}_{11} \mathrm{H}$, and $\mathrm{HC}_{13} \mathrm{H}$, which by symmetry are nonpolar, strong electronic spectra have been measured in the gas-phase by cavity ringdown absorption spectroscopy. Almost all of these molecules are good candidates for detection in space, and six in fact have now been detected in at least one astronomical source with large radio telescopes during the past two years. The laboratory astrophysics of the entire set is complete for the time being, in the sense that nearly all the rotational transitions of interest to radio astronomy can be calculated to a small fraction of $1 \mathrm{~km} \mathrm{sec}^{-1}$ in equivalent radial velocity. With powerful new radio facilities planned or under construction it would be surprising if many more could not eventually be found. For each of the four new symmetric chains detected in the visible, the wavelength of the origin band has been measured to high accuracy, permitting deliberate searches for these molecules in diffuse and translucent molecular clouds.
\end{abstract}

\section{Introduction}

In space as on Earth, the unique ability of carbon to form single and multiple bonds gives rise to a chemistry of considerable complexity and specificity. In rich interstellar and circumstellar sources, the majority of the nearly 100 polyatomic 
molecules so far discovered by rotational spectroscopy with radio telescopes are composed largely of carbon and possess linear or nearly linear heavyatom backbones. Somewhat paradoxically, most of these molecules are highly unsaturated even though hydrogen is far more abundant (by more than three orders of magnitude) than carbon through much of our Galaxy and others as well. Nearly all are also unsymmetrical for a reason of little fundamental importance: simply to provide the permanent electric dipole moment required for radio astronomical detection. It is widely believed that most astronomical molecules are produced via gas-phase ion-molecule reactions which occur rapidly even at the low density and ultra-cold temperatures in space. The abundance of carbon chains, in particular, can be attributed to the efficient formation of $\mathrm{C}^{+}$by the reaction $\mathrm{CO}+$ $\mathrm{He}^{+} \rightarrow \mathrm{C}^{+}+\mathrm{O}+\mathrm{He}$ (Klemperer 1995).

Because unsaturated carbon compounds are unstable at terrestrial densities, tending to explosively polymerize to amorphous carbon or soot, their laboratory detection and characterization has proved challenging, often lagging rather than preceding astronomical discovery. However, recently the application of Fourier transform microwave (FTM) and cavity ringdown absorption spectroscopies (CRS) to supersonic molecular beams has overcome this difficulty, yielding in our laboratory the detection of many new reactive molecules, including carbon chains and several exotic ring-chains and rings. The total number of new discoveries (Fig. 1) now stands at 56: fourteen are closed-shell polyynes, twenty-four are radicals, and eighteen are carbenes. Of these, 52 are polar molecules whose rotational spectra have been detected with our sensitive FTM spectrometer at centimeter wavelengths, and 4 are nonpolar molecules whose optical spectra have been detected with CRS at visible wavelengths.

All or nearly all of our 52 new polar molecules are good candidates for astronomical detection, and most probably exist in the interstellar or circumstellar gas at some level of abundance. The astronomically most interesting lines of each can be calculated to high precision from spectroscopic constants derived from the laboratory data. Five chains have already been detected in at least one astronomical source with radio telescopes in combination $\left(\mathrm{C}_{8} \mathrm{H}\right)$, or as a direct result of the laboratory work $\left(\mathrm{C}_{7} \mathrm{H}, \mathrm{HC}_{11} \mathrm{~N}, \mathrm{SiC}_{3}\right.$, and $\left.\mathrm{H}_{2} \mathrm{C}_{6}\right)$. The sixth molecule, protonated cyanoacetylene $\mathrm{HC}_{3} \mathrm{NH}^{+}$, the only ion we have so far detected, was first identified in the millimeter-wave band in TMC-1 prior to our work by Kawaguchi et al. (1994) on the basis of a laboratory infrared determination of its rotational constant.

The four non-polar carbon chain radicals, $\mathrm{HC}_{7} \mathrm{H}, \mathrm{HC}_{9} \mathrm{H}, \mathrm{HC}_{11} \mathrm{H}$, and $\mathrm{HC}_{13} \mathrm{H}$ detected by laser absorption spectroscopy, are also candidates for astronomical discovery. These chains are closely related in structure and composition to known astronomical molecules, and they possess intense electronic transitions in the visible. Furthermore, because the wavelengths of the $\Sigma-\Sigma$ bands of each radical are insensitive to the effective rotational temperature, the laboratory measurements provide extremely precise wavelengths for astronomical searches with optical telescopes.

For most of our new discoveries, the laboratory spectroscopy is now ahead of the observational astronomy, and astronomical searches, as they should to be efficient, can now be based on very precise data. The large number of laboratory detections also provides much new data for the next generation of radio 

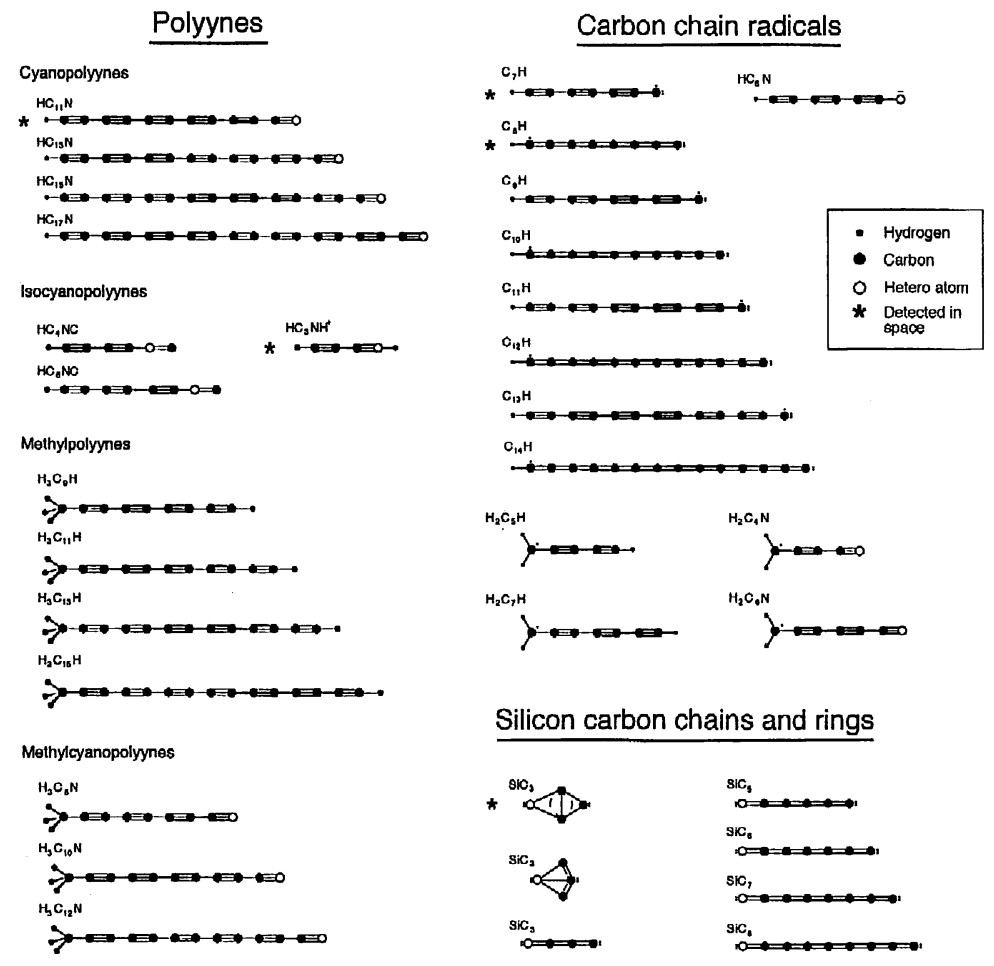

Silicon carbon chains and rings
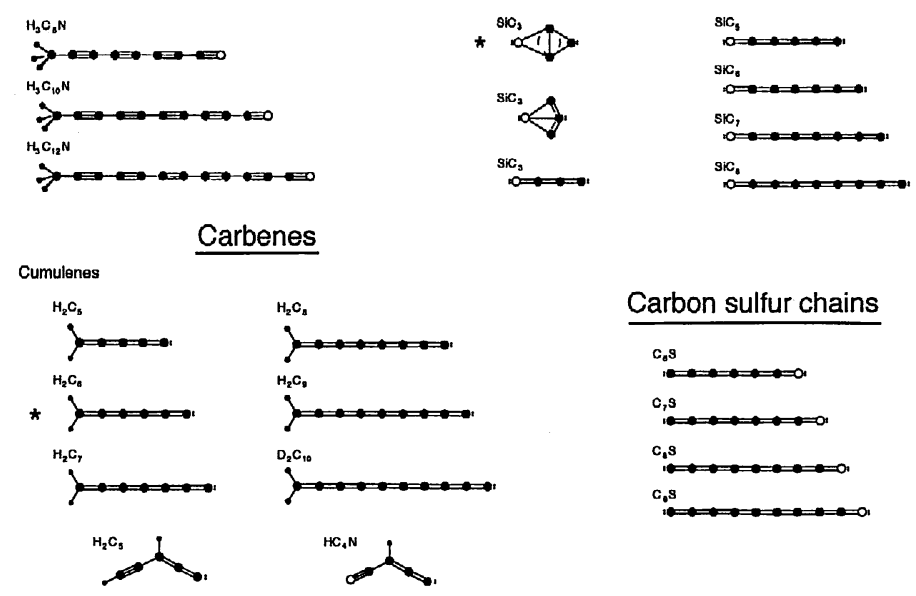

Ring-Chains

Symmetric carbon chain radicals
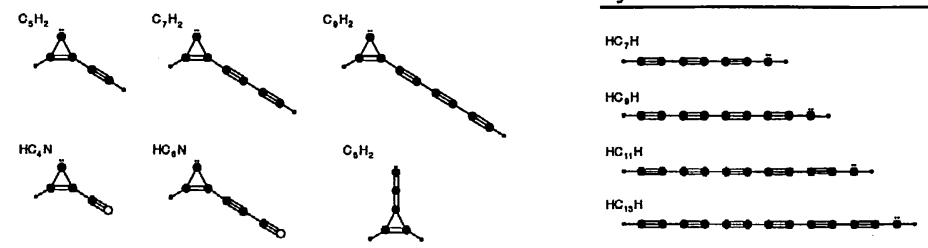

Figure 1. Fifty-six new carbon chains and rings. Open circles denote hetero atoms, either N, Si or S. The symmetric hydrocarbon radicals were previously detected in an inert matrix by Fulara et al. (1995). 
and optical astronomical investigations. Because of limited space it is not possible to give here a detailed description of our many newly discovered chains or much in the way of experimental details. Instead, I will briefly describe several examples that illustrate how laboratory astrophysical experiments can be applied to studies of known or postulated astronomical molecules, and discuss joint astronomical and laboratory investigations that have led to some of the recent astronomical discoveries.

\section{Recent Results}

\subsection{Long carbon chains: $\mathbf{H C}_{17} \mathbf{N}$}

The longest carbon chain so far detected in our laboratory is a large molecule even by the standards of known astronomical chains: $\mathrm{HC}_{17} \mathrm{~N}$ with 19 atoms and a molecular weight of $219 \mathrm{amu}$, nearly three times the molecular weight of glycinc, the simplest amino acid. Shown in Fig. 2 is an energy level diagram of the lower rotational levels of $\mathrm{HC}_{17} \mathrm{~N}$ and the transitions that have been measured, along with a sample line (McCarthy et al. 1998). Laboratory detection of longer cyanopolyynes beyond $\mathrm{HC}_{11} \mathrm{~N}$, the largest known interstellar molecule (Bell et al. 1997), and other long polyynes has been possible during the past two years with systematic improvements in production efficiency and in the detection sensitivity of our FTM spectrometer.

As found for other closed-shell chains, the rotational constants of the longer cyanopolyynes are remarkably well predicted by extrapolation in which the moments of inertia of the shorter chains in the sequence are fit to a third-order polynomial in chain length. The predicted constants agree with those measured to within a few parts in 100,000 for both $\mathrm{HC}_{15} \mathrm{~N}$ and $\mathrm{HC}_{17} \mathrm{~N}$, so that at $5 \mathrm{GHz}$ where $\mathrm{HC}_{17} \mathrm{~N}$ has its most intense rotational transitions in our $3 \mathrm{~K}$ molecular beam, a frequency search of $1 \mathrm{MHz}$ or less was required. The empirical predictions are at least an order of magnitude better than the best theoretical $a b$ initio predictions, which are no better than $0.1 \%$ in accuracy. The close agreement of extrapolation with experiment suggests that the moment of inertia of these long chains is quite insensitive to the structural details such as the rotation-vibration interaction which are important for small molecules.

The long cyanopolyynes here provide additional evidence that carbon chains under centrifugal distortion behave like thin classical rods, with a Young's modulus that is independent of length (Thaddeus et al. 1998). All carbon chains we have studied apparently distort under rotation in this simple way, regardless of either terminal groups or the low-lying vibrational structure of the individual molecules. One might expect such centrifugal distortion for long chains, but it is somewhat surprising that it holds for quite short ones, where quantum effects and the details of the vibrational structure of the molecule might be thought to be important.

The detection of $\mathrm{HC}_{17} \mathrm{~N}$ illustrates why it is important to push the sensitivity of our microwave spectrometer to its fundamental detection limit. As Fig. 3 shows, for longer acetylenic, cumulenic, and polyyne chains there is a considerably flatter decrement of line intensity with length than expected from the shorter members in the series. The lines of $\mathrm{HC}_{17} \mathrm{~N}$ in particular are nearly an order of magnitude stronger than predicted by the nearly constant decrement 


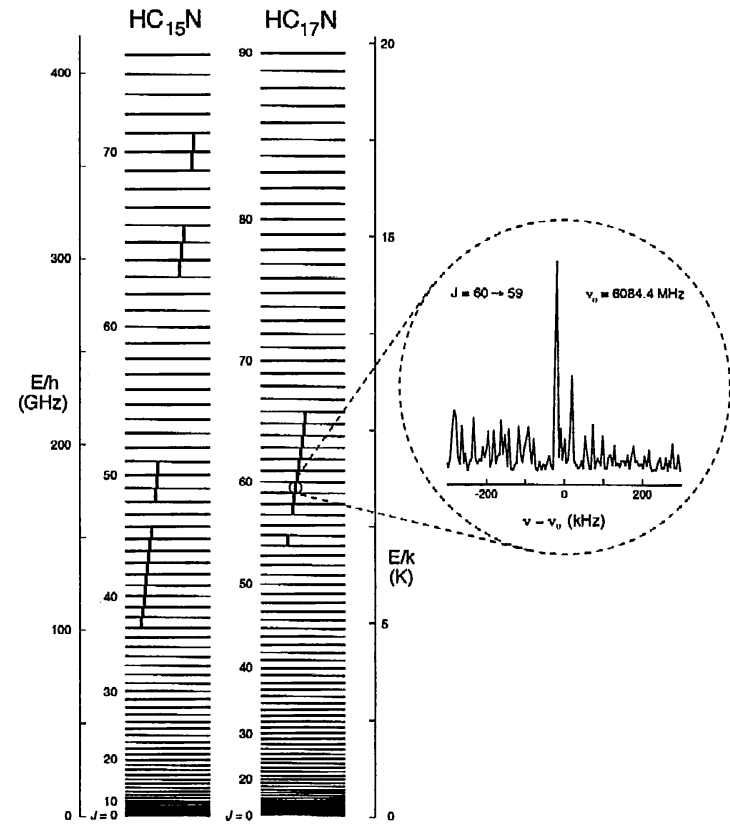

Figure 2. Lower rotational levels of $\mathrm{HC}_{15} \mathrm{~N}$ and $\mathrm{HC}_{17} \mathrm{~N}$ with observed transitions indicated (vertical bars). Insert: Sample line of $\mathrm{HC}_{17} \mathrm{~N}$ obtained with an integration time of $60 \mathrm{~min}$. The double peaked line shape is instrumental in origin - it results from the Doppler shift of the supersonic molecular beam relative to the two traveling waves that compose the confocal mode of the Fabry-Perot cavity.

in intensity observed for $\mathrm{HC}_{3} \mathrm{~N}$ to $\mathrm{HC}_{9} \mathrm{~N}$; in terms of abundance - the density of the molecule in our molecular beam - the decrement is almost zero. The longest cyanopolyynes are evidently more abundant and more easily detected than might have been expected, and still longer ones are probably within our grasp with a fairly modest improvement in sensitivity; little frequency search is probably required for $\mathrm{HC}_{21} \mathrm{~N}, \mathrm{HC}_{23} \mathrm{~N}$, etc., at most a few $\mathrm{MHz}$. In the interstellar gas, as in our molecular beam, one wonders if long chains may be similarly more abundant than extrapolation from short chains suggests.

Astronomical detection of large molecules such as $\mathrm{HC}_{17} \mathrm{~N}$ is not impossible. Larger and better telescopes, such as the Green Bank $100 \mathrm{~m}$ telescope (GBT) and the resurfaced Arecibo $305 \mathrm{~m}$ telescope, will soon be available in the 1$10 \mathrm{GHz}$ frequency range where the most intense astronomical lines of the larger molecules lie. Although many hours of integration were required to detect lines of $\mathrm{HC}_{11} \mathrm{~N}$, considerably less time may be necessary to detect other long chains in TMC-1 with these new telescopes. Single-dish and interferometric maps of $\mathrm{C}^{18} \mathrm{O}$, CCS, and CS (Langer et al. 1995) show that TMC-1 has a hierarchical structure ranging in size over two orders of magnitude: from large elliptical features (about $\left.10^{\prime}\right)$ to extremely small molecular cores or "clumps" $\left(10^{\prime \prime}\right)$. Since these small-scale structures may have the highest column density of molecules, and are not resolved with present single-dish telescopes (the beamwidth of the NRAO dish is about $6^{\prime}$ at $6 \mathrm{GHz}$ ), even larger telescopes with greater collecting areas and higher angular resolution should be considerably more efficient. If the source is unresolved, the $100 \mathrm{~m}$ GBT should be approximately five times more efficient than the NRAO $140 \mathrm{ft}$. telescope at $6 \mathrm{GHz}$.

Detection of the lowest frequency bending modes of long conjugated carbon chains may provide another opportunity to observe molecules as large as $\mathrm{HC}_{17} \mathrm{~N}$ in astronomical sources. The frequencies of the lowest bending mode of such 


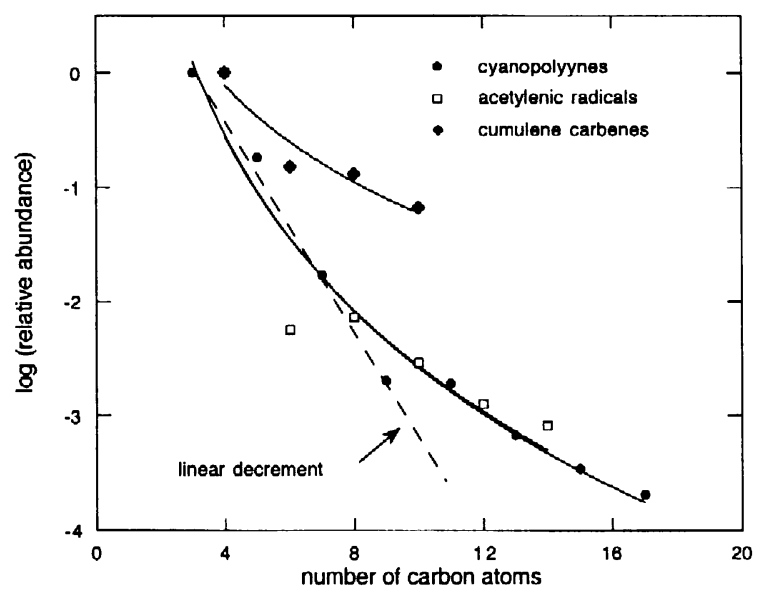

Figure 3. Relative abundances of the even-numbered acetylenic and cumulenic, and odd-numbered cyanopolyyne chains in the FTM spectrometer, as a function of the number of carbon atoms in the chain. The error bars are estimated $2 \sigma$ uncertainties. Relative abundances are obtained from line intensities by taking into account the dependence on the chain length of the rotational partition functions and dipole moments. The limit of the detection sensitivity is approximately that achieved in a total observation time of $3 \mathrm{hrs}$.

a chain can be estimated to an uncertainty of about $15 \%$ from a semi-classical calculation in which bending modes scale in frequency as the inverse square of the chain (Thaddeus et al. 1998). Extended to the two longest cyanopolyyne chains here, such scaling predicts $14 \mathrm{~cm}^{-1}(420 \mathrm{GHz})$ for $\mathrm{HC}_{15} \mathrm{~N}$ and $11 \mathrm{~cm}^{-1}$ $(330 \mathrm{GHz})$ for $\mathrm{HC}_{17} \mathrm{~N}$. Both frequencies are well within the range of existing radio telescopes such as the Caltech Submillimeter Telescope, and the atmosphere is fairly transparent at these wavelengths, but precise laboratory measurements of the vibrational transition frequencies and accurate calculations of the transition moments are clearly needed before undertaking such searches.

\subsection{Reactive rings: Rhomboidal $\mathrm{SiC}_{3}$}

A good example of the close interplay between laboratory astrophysics and astronomical observations is the recent discovery of rhomboidal $\mathrm{SiC}_{3}$, a planar highly polar ring molecule. $\mathrm{SiC}_{3}$ is the fifth and, in terms of size and mass, the largest cyclic compound so far detected in space with radio telescopes. With the exception of ethylene oxide, the only known stable interstellar ring, rhomboidal $\mathrm{SiC}_{3}$ and the three remaining cyclic compounds, $\mathrm{C}_{3} \mathrm{H}_{2}, \mathrm{C}_{3} \mathrm{H}$, and SiCC, are all highly reactive rings generally unfamiliar to the laboratory chemist or physicist. In the laboratory, as in the interstellar gas, the detection of reactive rings has lagged behind detection of chains of similar size and composition in part because cyclic molecules lack simple harmonically-related rotational transitions that are readily spotted. As a result, it is perhaps not surprising that laboratory spec- 
troscopy has preceded astronomical discovery for all five ring molecules. The detection of new reactive rings is worth pursuing because these molecules provide quite novel information on the rich and largely unexplored transition of carbon from chains to the two-dimensional graphitic lattice.

Rotational transitions of rhomboidal $\mathrm{SiC}_{3}$ and its rare isotopic species were first observed in the centimeter-wave band (McCarthy, Apponi, \& Thaddeus 1999) with our FTM spectrometer in a pulsed discharge with a dilute mixture of diacetylene and silane - an efficient combination which yields high concentrations of $\mathrm{SiC}_{3}$ and longer linear silicon-carbides up to $\mathrm{SiC}_{8}$. At most four rotational transitions (Fig. 4) of each isotopic species lie within the frequency range of this spectrometer, but this number alone is sufficient to unambiguously establish the existence of this exotic ring, and to allow a precise determination of its geometry. If $\mathrm{SiC}_{3}$ were strictly planar and rigid, two parameters would suffice to determine the entire spectrum: three moments of inertia constrained by the Pythagorean theorem, but to describe the actual deformable molecule to the accuracy needed to conduct a radio astronomical search requires the thrce rotational constants and several centrifugal distortion constants. Using our freespace millimeter-wave absorption spectrometer, the millimeter-wave spectrum of $\mathrm{SiC}_{3}$ was subsequently measured (Apponi et al. 1999b) to within a few parts in $10^{7}$ up to $300 \mathrm{GHz}$, and six leading centrifugal distortion constants were determined to high precision.

On the basis of the laboratory data, a total of 11 millimeter-wave rotational transitions of $\mathrm{SiC}_{3}$ have now been detected in the circumstellar shell of the evolved carbon star IRC+10216 using the $12 \mathrm{~m}$ NRAO telescope on Kitt Peak (Apponi et al. 1999a). The lines are fairly weak $(\sim 5 \mathrm{mK})$, but all have a mean radial velocity of $-26(3) \mathrm{km} \mathrm{s}^{-1}$, precisely that found for most other molecules in this source. The rotational excitation of $\mathrm{SiC}_{3}$ is similar to $\mathrm{SiCC}$, with a low rotational temperature within the $K$-stacks of $10-20 \mathrm{~K}$, and a high rotational temperature across the $K$-stacks of roughly $50 \mathrm{~K}$, which is probably fairly close to the kinetic temperature of $\mathrm{H}_{2}$ gas, the main constituent of the shell. The column density of $\mathrm{SiC}_{3}$ is calculated to be $4.3 \times 10^{12} \mathrm{~cm}^{-2}$ assuming a shell diameter of $60^{\prime \prime}$ - perhaps $50 \%$ larger than that of $\mathrm{SiC}_{4}$.

Photochemical models of IRC+10216 by Glassgold \& Mamon (1992) predict that, as observed, $\mathrm{SiC}_{3}$ and $\mathrm{SiC}_{4}$ are more than two orders of magnitude less abundant than either $\mathrm{SiC}$ and $\mathrm{SiCC}$. They also predict that some $\mathrm{SiC}_{3}$ will be produced by the photodissociation of $\mathrm{SiC}_{4}$. The implication is that the total amount of $\mathrm{SiC}_{3}$ cannot exceed that of $\mathrm{SiC}_{4}$. If $\mathrm{SiC}_{3}$ is found to be more abundant than $\mathrm{SiC}_{4}$, however, other reactions may be required to explain the observed abundance. To clarify the formation of $\mathrm{SiC}_{3}$ and to better understand if rhomboidal $\mathrm{SiC}_{3}$ is actually produced via photodissociation of linear $\mathrm{SiC}_{4}$, it would be desirable to detect additional $\mathrm{SiC}_{3}$ isomers in IRC+10216, and compare their abundances. Two other low-lying, fairly polar $\mathrm{SiC}_{3}$ isomers, a triplet chain and second rhomboidal isomer, have now been detected in this laboratory. Astronomical searches for both are underway.

\subsection{Non-polar chains: Linear triplet $\mathrm{HC}_{7} \mathrm{H}$ and $\mathrm{HC}_{9} \mathrm{H}$}

Although the microwave spectroscopy of the 52 polar molecules shown in Fig. 1 is complete in the sense that the identity of each molecule is secure and precise 


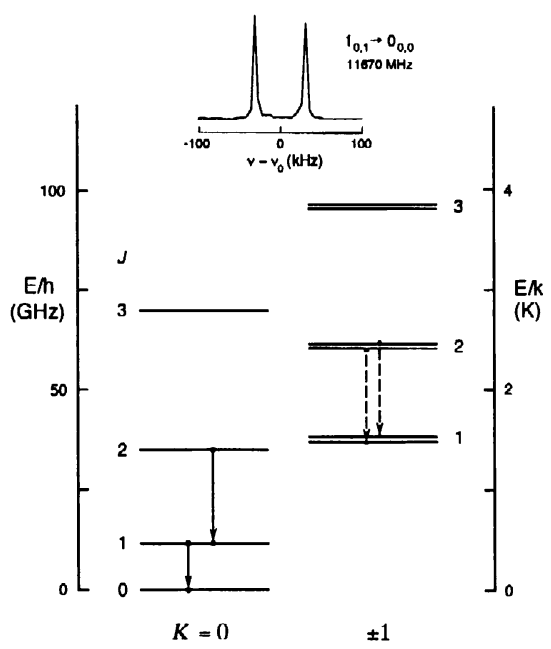

Figure 4. Energy level diagram of $\mathrm{SiC}_{3}$ showing the lines measured in the normal and rare isotopic species. Solid arrows indicate rotational transitions measured in the $K=0$ ladder for all six isotopic species; dashed arrows indicate additional transitions measured in $K= \pm 1$ ladder for the two ${ }^{13} \mathrm{C}$ isotopic species where one or both equivalent carbon atoms is replaced with ${ }^{13} \mathrm{C}$. Insert: spectrum of the $1_{0,1} \rightarrow 0_{0,0}$ rotational transitions of $\mathrm{SiC}_{3}$ at $11,670 \mathrm{MHz}$, observed with a single gas pulse and an integration time of $0.2 \mathrm{msec}$.

spectroscopic constants for the ground state of each have been determined, the electronic spectra of most of these molecules remains a complete blank. Under the right conditions, however, electronic spectroscopy is generally much more sensitive in the laboratory than rotational spectroscopy. The red and violet bands of CN, for example, were first identified in 1932 (Jenkins, Roots, \& Mulliken), but it was not until 1977 - a period spanning more than 40 years - that the rotational spectrum of this important radical was observed by millimeterwave absorption spectroscopy (Dixon \& Woods 1977). Most of our 48 new molecules have conjugated structures, similar in their properties to organic dyes, with similarly strong electronic transitions that make them good candidates for the carriers of the diffuse interstellar bands. Since the specific abundance of each molecule in our molecular beam is known accurately from the microwave experiments, we now know that most are produced with adequate abundance for laser detection.

Using the same discharge nozzle source which was used successfully in our FTM spectrometer, we have recently undertaken laser optical studies on our microwave carbon chains and rings. Very promising results (Ball, McCarthy, \& Thaddeus 1999) were achieved shortly after this spectrometer was constructed, when strong optical spectra of two carbon chain radicals $\mathrm{HC}_{7} \mathrm{H}$ and $\mathrm{HC}_{9} \mathrm{H}$ where detected by CRS (Fig. 5). For both radicals, which are non-polar by symmetry, the wavelengths of the strongest bands have been measured to an accuracy of 


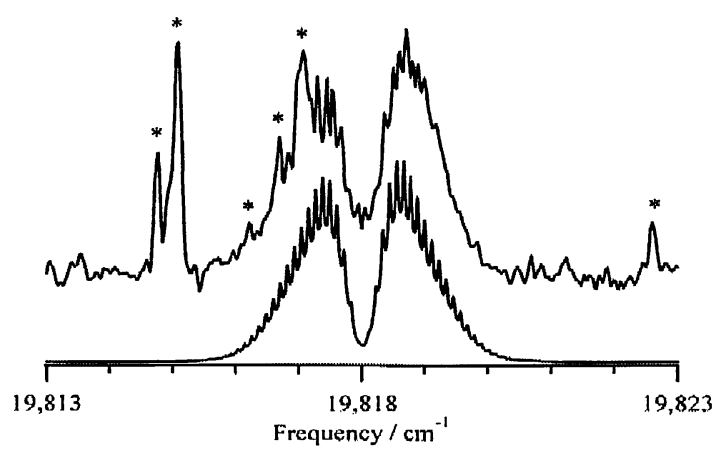

Figure 5. Measured and simulated spectra (upper and lower traces, respectively) of the origin band of $\mathrm{HC}_{7} \mathrm{H}$, showing $P$ and $R$ branches characteristic of a $\Sigma-\Sigma$ vibronic band. The simulated spectrum shows that the individual rotational lines are only partially resolved because of broadening due to internal conversion. Asterisks indicate unrelated lines of $\mathrm{C}_{2}$.

about 1 part in $10^{6}$, and at least one vibronic band of each chain has also been measured to about the same level of accuracy. Because the origin bands of the two radicals have nearly the same intensity, and because the wavelengths of the strongest bands of each member in this homologous series are known fairly precisely from matrix spectra (Fulara et al. 1995), it has now been possible to detect the gas-phase spectra of the next two odd-numbered chains $\mathrm{HC}_{9} \mathrm{H}$ and $\mathrm{HC}_{11} \mathrm{H}$. For all four molecules, internal conversion seems to be much more rapid than radiative emission, and fluorescence is not observed. For $\mathrm{HC}_{9} \mathrm{H}$, the origin band is close in wavelength to a weak diffuse band at $\lambda 5818$, but recent astronomical observations at the Fort Apache Point Observatory by McCall, York, \& Oka (1999, private communication) conclude that the difference in wavelength between the two is too large for $\mathrm{HC}_{9} \mathrm{H}$ to be the carrier of $\lambda 5818$.

Even though there is yet no evidence that our symmetric hydrocarbon radicals are carriers of the DIBs, these chains remain excellent candidates for astronomical detection in both the diffuse gas and in cool stellar atmospheres or circumstellar shells, because of their structural similarity to known interstellar molecules. Because all four chains have $\Sigma-\Sigma$ transitions, it is worth emphasizing that extremely precise wavelengths for each band can be determined from the laboratory data. High accuracy is possible because these transitions lack both spin-orbit splitting and red-shaded $R$-heads which tend to shift the wavelengths of other electronic transitions by $0.05 \mathrm{~nm}$ or more depending on the rotational temperature either in the molecular beam or along different lines of sight in space. Since neither complication exists here, the band contours of the $\Sigma-\Sigma$ transitions are insensitive to rotational temperature, and, as a result, the wavelengths of the origin bands can be determined about an order of magnitude more precisely than most $\Pi-\Pi$ transitions. If transitions of the symmetric chains can eventually be found in space, confirmation of the assignment would then come from the characteristic $P$ - and $R$-branches in Fig. 5 , or by detection of their somewhat weaker vibronic bands. Our recent experiments imply that the optical spectra of most of the rest of the molecules shown in Fig. 1 may now be 
possible. By extension, the same may be true for other known and postulated astronomical molecules; if so, it should eventually be possible to return to the normal paradigm where optical detection either precedes or occurs soon after radio detection.

Acknowledgments. I thank my collaborators P. Thaddeus, C. A. Gottlieb, A. J. Apponi, and C. D. Ball for helpful discussions. The assistance of other scientists who are coauthors on recent papers is also gratefully acknowledged.

\section{References}

Apponi, A.J., McCarthy, M.C., Gottlieb, C.A., \& Thaddeus, P. 1999a, ApJ, $516, \mathrm{~L} 103$

1999b, J. Chem. Phys., 111, 3911

Ball, C.D., McCarthy, M.C., \& Thaddeus, P. 1999, ApJ, 523, L89

Bell, M.B., et al. 1997, ApJ, 483, L61

Dixon, T.A. \& Woods, R.C. 1977, J. Chem. Phys., 67, 3956

Fulara, J., Freivogel, P., Forney, D., \& Maier, J.P. 1995, J. Chem. Phys., 103, 8805

Glassgold, A.E. \& Mamon, G.A. 1992, in Chemistry and Spectroscopy of Interstellar Molecules, eds. D.K. Bohme et al. (Tokyo: U. Tokyo Press), 261

Jenkins, F.A., Roots, Y.K., \& Mulliken, R.S. 1932, Physic. Rev., 39, 16

Kawaguchi, K., et al. 1994, ApJ, 420, L95

Klemperer, W. 1995, Annu. Rev. Phys. Chem., 46, 1

Langer, W.D., et al. 1995, ApJ, 453, 293

McCarthy, M.C., Apponi, A.J., \& Thaddeus, P. 1999, J. Chem. Phys., 110, 10645 McCarthy, M.C., et al. 1998, ApJ, 494, L231

Thaddeus, P., McCarthy, M.C., Travers, M.J., Gottlieb, C.A., \& Chen, W. 1998, Faraday Discuss., 109, 121

\section{Discussion}

S. Saito: You showed the identification of geometrical isomers having the same elemental composition. Is it possible to estimate or determine their energy difference in the laboratory? The energy difference is important in determining their abundances in space. Are there any good ab initio predictions for the difference?

M. C. McCarthy: We cannot determine the energy difference between isomers from our laboratory data. H.F. Schaefer and co-workers, however, have performed high level theoretical calculations on the isomer of $\mathrm{SiC}_{3}$, and determined their relative energies. They find that the ground state of $\mathrm{SiC}_{3}$ is a prolate rhombad, and, lying only $5 \mathrm{kcal} \mathrm{mol}^{-1}$ above ground, there are two isoenergetic forms, a second oblate rhombad and a linear triplet chain. All three of these isomers have now been identified and characterized in our laboratory: their abundances in our molecular beam are consistent with the energies predicted by Schaefer et al. 